\title{
Response to PD-1 Blockade in Microsatellite Stable Metastatic Colorectal Cancer Harboring a POLE Mutation
}

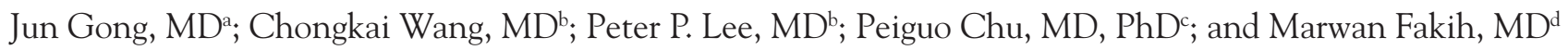

\begin{abstract}
Recent clinical evidence has demonstrated that microsatellite instability (MSI) or defective mismatch repair (MMR) and high tumor mutational load can predict response to the programmed cell death 1 (PD-1) receptor inhibitor pembrolizumab in metastatic colorectal cancer (mCRC). Mutations in polymerase $\varepsilon(P O L E)$, a DNA polymerase involved in DNA replication and repair, contribute to an ultramutated but microsatellite stable (MSS) phenotype in colorectal tumors that is uniquely distinct from MSI tumors. This report presents the first case in the literature describing a clinical response to pembrolizumab in an 81-year-old man with treatment-refractory mCRC characterized by an MSS phenotype and POLE mutation identified on genomic profiling by next-generation sequencing. On tumor immunostaining, a large amount of CD8-positive tumor infiltrating lymphocytes (TILs) were present, with $>90 \%$ of these expressing PD-1. More than $99 \%$ of PD-L1 expression was identified on nontumor cells in the tumor microenvironment that were close to the PD-1-positive CD8 TILs. mCRC tumors harboring POLE mutations represent a hypermutated phenotype that may predict response to anti-PD-1 therapy.
\end{abstract}

J Natl Compr Canc Netw 2017;15(2):142-147

Colorectal cancer $(\mathrm{CRC})$ represents the third-leading cause of cancer death in both men and women in the United States, with an estimated 49,190 deaths in 2016. ${ }^{1}$ The treatment landscape for metastatic CRC (mCRC) is becoming more molecularly driven. The addition of epidermal growth factor receptor (EGFR)-targeting agents to conventional cytotoxic therapy based on RAS and BRAF mutation status in mCRC serves as a recent example of selecting optimal therapy according to patient genomic profiles and molecular phenotypes. ${ }^{2}$ This report presents a case in which the programmed cell death 1 (PD-1) receptor inhibitor pembrolizumab was offered based on comprehensive genomic profiling, which identified a polymerase $\varepsilon$ (POLE) mutation associated with an ultramutated tumor in a patient with KRAS-mutated, microsatellite stable (MSS) metastatic colon adenocarcinoma.

From the aDepartment of Medical Oncology, ${ }^{\text {bDepartment }}$ of ImmunoOncology, Beckman Research Institute, 'Department of Anatomic Pathology, Beckman Research Institute, and ${ }^{\mathrm{d}}$ Medical Oncology and Experimental Therapeutics, City of Hope Comprehensive Cancer Center, Duarte, California.

Submitted September 13, 2016; accepted for publication November 9, 2016.

\section{Case Presentation}

An 81-year-old Hispanic man was referred to our gastrointestinal medical oncology clinic for recurrent colon adenocarcinoma. He was initially diagnosed with stage II (pathologic stage T3NOMO) right-sided colon adenocarcinoma and underwent a right hemicolectomy with negative margins. No adjuvant chemotherapy was given, and he was followed via observation. Nearly 3 years later, he experienced a recurrence with a large, high-grade obstructing mass near the hepatic flexure at the previous anastomotic site. Colonoscopy with biopsy confirmed a moderately differentiated adenocarcinoma. His carcinoembryonic antigen (CEA) level was elevated at $23 \mathrm{ng} / \mathrm{mL}$. CT scan confirmed an $8 \times 5.6-\mathrm{cm}$ anastomotic mass with extension into the inferior right lobe of the liver, gallbladder, and right perirenal fat; multiple enlarged regional mesenteric lymph nodes; and enlarged

The authors have disclosed that they have no financial interests, arrangements, affiliations, or commercial interests with the manufacturers of any products discussed in this article or their competitors.

Correspondence: Marwan Fakih, MD, Medical Oncology and Experimental

Therapeutics, City of Hope Comprehensive Cancer Center, Building 51,

Room 127, 1500 East Duarte Street, Duarte, CA 91010.

E-mail:mfakih@coh.org 
nonregional retroperitoneal lymph nodes. His recurrent disease was not deemed curatively resectable.

Microsatellite instability (MSI) testing via polymerase chain reaction analysis showed that the recurrent tumor was MSS. Furthermore, next-generation sequencing (NGS), using a CLIA-certified Onco48 genomic analysis (Clinical Molecular Diagnostics Laboratory, City of Hope National Medical Center, Duarte, CA) determined the presence of 2 rare KRAS mutations, $\mathrm{N} 116 \mathrm{H}$ and $\mathrm{N} 116 \mathrm{~T}$, at codon 116. The patient was subsequently treated with firstline 5-FU, leucovorin, and oxaliplatin (FOLFOX). Surveillance imaging after 4 cycles of FOLFOX identified that the anastomotic mass had decreased to 3.4 $\mathrm{x} 4.7 \mathrm{~cm}$ with cavitation and that several mesenteric lymph nodes had also decreased in size. Because of significant fatigue, the patient was transitioned to maintenance 5-FU, leucovorin, and bevacizumab starting from cycle 5 . He received an additional 11 cycles of maintenance 5-FU, leucovorin, and bevacizumab (16 cycles overall) followed by disease progression prompting transition to $5-\mathrm{FU}$, leucovorin, and irinotecan (FOLFIRI) with bevacizumab. Unfortunately, the patient experienced protracted grade 3 fatigue with FOLFIRI with a worsening performance status, and opted to discontinue this regimen. Surveillance CT imaging before cycle 1 of FOLFIRI had confirmed further disease progression in the hepatic flexure mass.

At this point, an expanded genomic analysis via FoundationOne (Foundation Medicine, Inc., Cambridge, MA) was performed on his archival tumor tissue. Molecular profiling identified a hypermutated tumor profile encompassing 100 total genomic alterations with a tumor mutational burden (TMB) of 122 mutations per megabase (Mb); alterations in POLE $E^{\mathrm{V} 411 \mathrm{~L}}$ and $R A F 1 R^{256 \mathrm{~S}}$ were also identified. Because of the patient's poor tolerance to conventional cytotoxic agents and comprehensive genomic analysis revealing a hypermutated tumor bearing a POLE mutation, we initiated treatment with pembrolizumab, a human monoclonal IgG4 antibody targeting PD-1. He received 3 cycles of pembrolizumab intravenously at $200 \mathrm{mg}$ every 3 weeks and experienced an improvement in his performance status and resolution of fatigue. His CEA level decreased from 23 to $1.3 \mathrm{ng} / \mathrm{mL}$ by cycle 6 and remains normal at the time of this report. Surveillance CT scans showed significant and persistent reduction in tumor burden at the site of anastomotic recurrence and a decrease in mesenteric lymphadenopathy after cycles 3 and 6 (Figure 1). At the time of writing, the patient had completed 8 cycles of pembrolizumab with ongoing clinical benefits and no associated toxicity.

Multispectral fluorescent immunohistochemistry was performed on the patient's archival colon tumor at the site of anastomotic recurrence to further investigate the degree of immune response and immune checkpoint upregulation. Immunostaining identified a large proportion of CD8-positive tumor-infiltrating lymphocytes (TILs), of which $>90 \%$ were PD-1-positive (Figure 2). Additionally, $>99 \%$ of programmed deathligand 1 (PD-L1) expression occurred in nontumor cells, particularly in the tumor microenvironment close to the PD-1-positive CD8 TILs.

\section{Discussion}

The search for genetic alterations of potential therapeutic importance in CRC continues to gain momentum with the development of comprehensive genomic profiling using techniques such as NGS with high-throughput functionality. The Cancer Genome Atlas (TCGA) Network recently performed a comprehensive molecular characterization of 224 colorectal tumors $^{3}$ and identified several molecular alterations considered targetable, including mediators of aberrant WNT, RAS, and PI3K signaling, such as ERRB2, ERRB3, MEK, AKT, MTOR, IGF2, and IGFR. Of note, $16 \%$ of colorectal tumors were found to be hypermutated and more frequently located on the right colon. Of these hypermutated tumors, three-quarters had high MSI (MSI-H).

The significance of MSI in identifying molecular profile-driven therapies in mCRC was recently elaborated in a phase II trial enrolling 41 patients with treatment-refractory metastatic cancers stratified into 3 cohorts: mismatch repair (MMR)-deficient colorectal tumors (cohort A), MMR-proficient colorectal tumors (cohort B), and MMR-deficient noncolorectal tumors (cohort C). ${ }^{4}$ Treatment with the anti-PD-1 immune checkpoint inhibitor pembrolizumab (10 mg/kg intravenously every 14 days) showed significantly improved immune-related objective response rates and survival for cohorts $\mathrm{A}$ and C compared with cohort B. Hazard ratios (HRs) for disease progression or death (HR, 0.10;95\% CI, 0.03-0.37; P<.001) and death (HR, 0.22; 95\% 
Gong et al

CI, 0.05-1.00; $P=.05)$ significantly favored MMRdeficient CRC compared with MMR-proficient CRC. Genomic analysis identified that MMR-deficient tumors had a significantly higher mutational load compared with MMR-proficient tumors, and was associated with prolonged progression-free survival $(P=.02)$. Findings from this pivotal study and others $^{5,6}$ have since corroborated the concept that MSI caused by defective MMR and high TMB can predict response to pembrolizumab in mCRC. Additionally, nivolumab (anti-PD-1 monoclonal IgG4 antibody) with or without ipilimumab (anti-cytotoxic T-lymphocyte-associated protein 4 [antiCTLA-4] antibody) has also recently demonstrated benefit in previously treated MSI-H mCRCs. ${ }^{7}$

Interestingly, one-quarter of hypermutated tumors in the CRC data set from TCGA were not MSI$\mathrm{H}$ but carried somatic mutations in MMR genes or POLE. ${ }^{3}$ POLE and polymerase $\delta$ (POLD1) are DNA proofreading enzymes in which mutations in the exonuclease domain, particularly hot spot residues P286, V411, and S459 of POLE, predispose to extremely high rates of base substitution mutations. ${ }^{8,9}$ Germline and somatic mutations in POLE and POLD1 contribute to an ultramutated but MSS phenotype in CRCs and endometrial cancers. ${ }^{9}$ In CRC retrospective cohorts, somatic POLE mutations were identified in $1 \%$ of CRC cases, although these mutations were more frequently found in younger (as high as $9.8 \%$ of cases) and male patients, right-sided colorectal tumors, and earlier stages of disease. ${ }^{10,11}$ Furthermore, POLE-mutated CRC was associated with an excellent prognosis, had among the highest mutational burden, and was mutually exclusive with defective MMR and MSS tumors. ${ }^{11,12}$ POLE-induced mutations are highly immunogenic and capable of eliciting an antitumor immune response putatively caused by enrichment of mutation-associated neoantigens. ${ }^{13,14}$ To counteract the increase in infiltrating immune cells, POLE-mutated and MSI tumors (hypermutated phenotype) upregulate expression of immune checkpoints, including PD-1, PD-L1, and CTLA-4, as evidenced in our case; this phenomenon renders these tumors excellent candidates for checkpoint inhibitors. ${ }^{15,16}$

In a cohort of 34 patients with previously treated and treatment-naïve advanced or metastatic nonsmall cell lung cancer, 2 patients with deleterious POLE mutations experienced durable responses
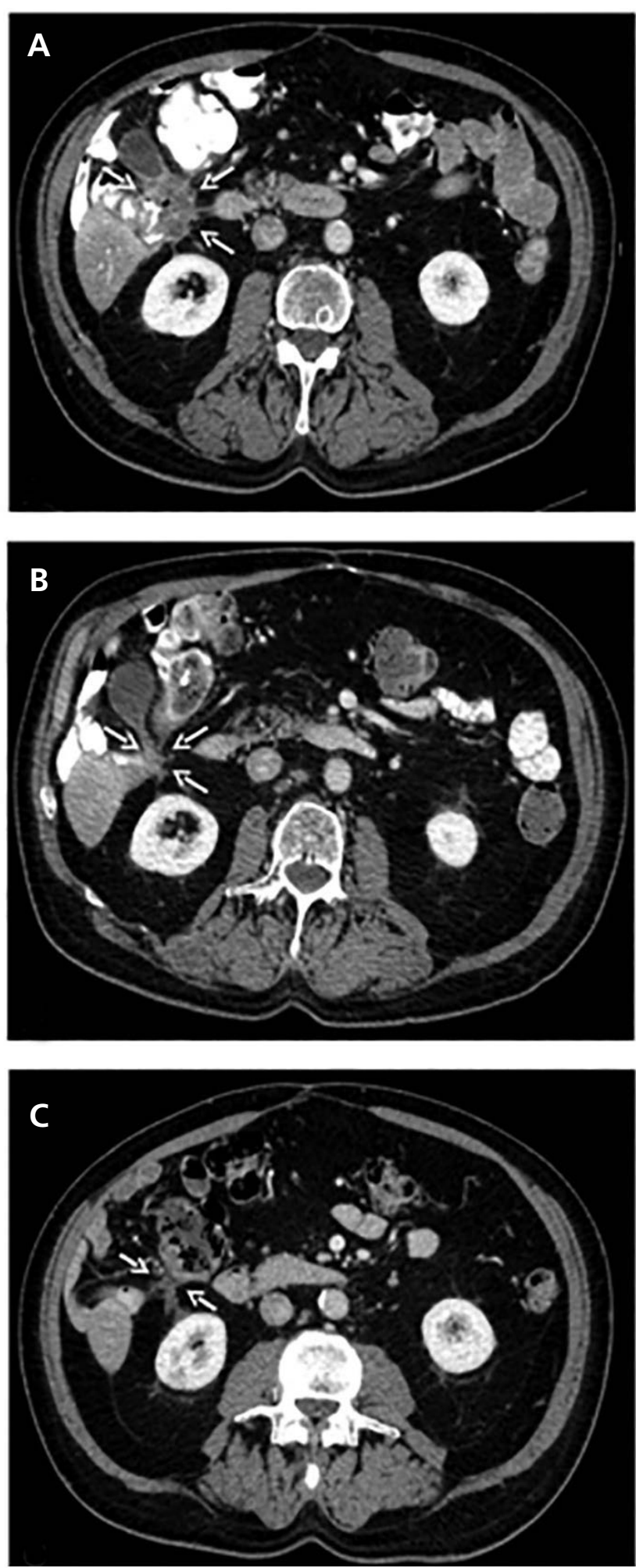

Figure 1. Significant response in a POLE-mutated recurrent colon adenocarcinoma arising near the hepatic flexure (white arrows) with extension into the liver, gallbladder, and right perirenal fat (A) before, (B) after 3 cycles, and (C) after 6 cycles of the PD-1 inhibitor pembrolizumab at $200 \mathrm{mg}$ every 3 weeks. 

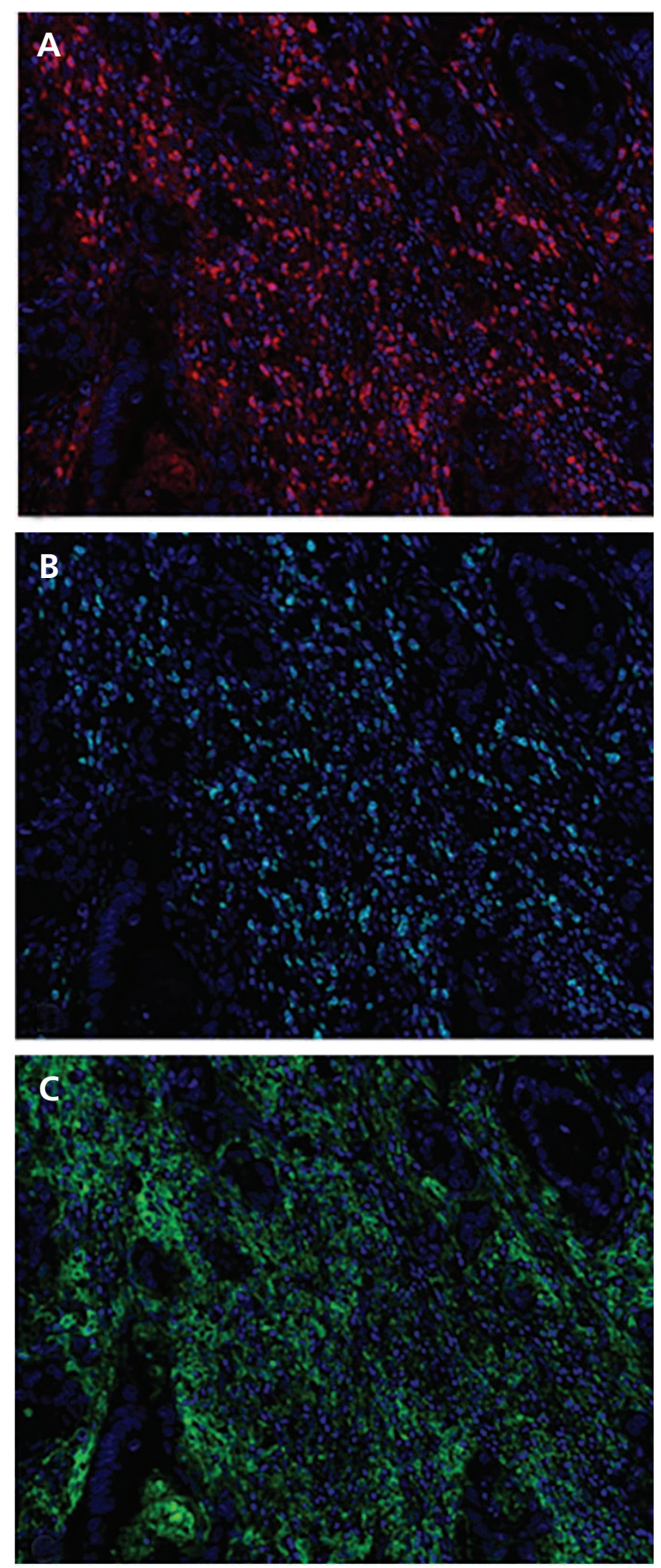

Figure 2. Multispectral fluorescent immunohistochemistry with a panel including CD8, programmed cell death 1 (PD-1), and programmed death-ligand 1 (PD-L1) in a POLE-mutated recurrent colon adenocarcinoma arising near the hepatic flexure identified a large population of (A) CD8-positive tumor infiltrating lymphocytes (TILs; red). (B) PD-1 expression (cyan) was present in $>90 \%$ of these CD8-positive T-cells. (C) More than $99 \%$ of PD-L1 was expressed (green) on nontumor cells in the tumor microenvironment that were in proximity to the PD-1positive CD8 TILs. Slides were scanned using the PerkinElmer Vectra and images were analyzed using the inForm software (PerkinElmer, Hopkinton, MA). (progression-free survival, 8 and 14 months, both ongoing) to treatment with pembrolizumab, $10 \mathrm{mg} /$ kg every 2 to 3 weeks. ${ }^{17}$ These tumors also had the highest nonsynonymous mutation burden in the cohort. A separate case report involving a 53-year-old woman with recurrent and metastatic high-grade endometrial adenocarcinoma (endometrioid type) harboring POLE $\mathrm{V}^{\mathrm{V} 11 \mathrm{~L}}$ and POLE $E^{\mathrm{R} 114}$ mutations experienced a partial response at 8 weeks with pembrolizumab, $10 \mathrm{mg} / \mathrm{kg}$ every 2 weeks, that has since been sustained for more than 14 months. ${ }^{18}$ Remarkable clinical response has also been seen in a case of heavily pretreated and hypermutated metastatic endometrial cancer harboring a POLE ${ }^{\mathrm{P} 286 \mathrm{R}}$ mutation treated with nivolumab, $3 \mathrm{mg} / \mathrm{kg}$ every 2 weeks. ${ }^{19} \mathrm{We}$ have investigated a database of 210 patients with mCRC using FoundationOne analysis under Institutional Review Board Protocol 14361 at our institution. Notably, a second patient (51-year-old man) with RAS/BRAF wild-type, HER2-amplified mCRC carrying a POLE ${ }^{\mathrm{R} 46 \mathrm{Q}}$ mutation has been identified. Interestingly, a concurrent POLD $1^{\mathrm{G} 321 \mathrm{~S}}$ mutation was also present. In contrast to the hypermutated status in our case report (TMB of 122 mutations per $\mathrm{Mb}$ ), this second case of POLE mutation had only $17 \mathrm{ge}$ nomic alterations and a TMB of 4 mutations per $\mathrm{Mb}$, although the mutation was not at a hot spot residue known to predispose to high mutational burden. Furthermore, we have identified 5 patients with MSI$\mathrm{H}$ mCRC demonstrating total genomic alterations ranging from 36 to 68 and $\mathrm{TMB}$ of $16,31,33,42$, and 73 mutations per $\mathrm{Mb}$.

Mutational burden has been shown to be significantly higher in POLE-mutated tumors than in MSI tumors. ${ }^{15,18}$ In a cohort with endometrial cancer, POLE-mutated tumors had a significantly higher mutational burden followed by MSI tumors and was lowest in MSS tumors. ${ }^{15}$ In an exploration of an early-stage endometrioid endometrial cancer data set from TCGA, POLE-mutated tumors demonstrated higher levels of immune checkpoints, including PDL1 and PD-L2, compared with MSI or MSS tumors. ${ }^{18}$ Higher levels of T-cell markers, including CD8A, PD-1, and CTLA-4, as well as higher proportions of CD8-positive T-cells, T-follicular helper cells, M1 macrophages, and activated natural killer cells were seen in POLE-mutated tumors compared with MSS tumors, with MSI tumors having intermediate levels. Similarly, POLE-mutated tumors had a higher 
extent of histologic TILs than MSS tumors, with MSI tumors showing an intermediate phenotype. Interestingly, a separate study exploring early-stage endometrioid endometrial cancers similarly identified significantly higher numbers of CD3-positive and CD8-positive TILs, and more frequent PD-L1 expression in POLE-mutated tumors and MSI tumors compared with MSS tumors. ${ }^{15}$ However, there were no significant differences between POLE-mutated and MSI tumors in the number of CD3-positive and CD8-positive TILs and PD-L1 expression. In a large cohort of predominantly early- to locally advanced-stage CRC, POLE-mutated tumors showed significantly increased CD8-positive TILs and a 2to 5-fold increased expression of cytotoxic T-cell markers and effector cytokines than MSS tumors. ${ }^{11}$ Notably, POLE-mutated CRC also had significantly greater expression of immune checkpoints CTLA-4, PD-1, and PD-L1 compared with MSS tumors; the degree of immune response upregulation was similar to that seen in MSI tumors. ${ }^{11,20}$ Data on the immune signature of $\mathrm{mCRC}$ harboring POLE mutations com- pared with MSI-H mCRC are unfortunately lacking. Our findings also suggest that significant heterogeneity may exist in the mutation load of tumors harboring POLE mutations depending on occurrence of mutations at hot spot residues known to predispose to high rates of mutations. This finding may have clinical significance in view of the known correlation between response to checkpoint inhibitors and mutational load.

We offered treatment with pembrolizumab based on the FoundationOne NGS results revealing a POLE ${ }^{\mathrm{V} 411 \mathrm{~L}}$ mutation in our hypermutated patient with previously treated and MSS recurrent colon adenocarcinoma. To our knowledge, this is the first case describing response to pembrolizumab in an mCRC tumor with a POLE mutation. Our findings are hypothesis-generating and warrant further investigation of checkpoint inhibition in POLE-mutated mCRCs, ideally in prospective settings. Focus should be on the potential differential effects of POLE mutations on proofreading function and resultant mutational burden, and their relationship to response

\section{Table 1. Tumor Molecular Profiles of Known or Potential Significance}

\begin{tabular}{|c|c|c|c|}
\hline Molecular Phenotype & Function & Source & Reference \\
\hline $\begin{array}{l}\text { Lynch syndrome (germline } \\
\text { mutations in } M L H 1, M S H 2 \text {, } \\
\text { MSH6, or PMS2) }\end{array}$ & DNA MMR & $\begin{array}{l}\text { Phase II cohort of } 11 \text { patients with Lynch syndrome by } \\
\text { clinical criteria; PCR showed increased mutational load } \\
\text { by WES }\end{array}$ & 4,22 \\
\hline $\begin{array}{l}\text { Sporadic mutations in } M L H 1 \text {, } \\
\text { MLH3, MSH2, MSH3, MSH6, } \\
\text { and PMS2 }\end{array}$ & DNA MMR & $\begin{array}{l}\text { WGS showed that among } 30 \text { hypermutated tumors, } 19 \\
\text { had MLH1 methylation }\end{array}$ & 3 \\
\hline CIMP & $\begin{array}{l}\text { DNA promoter methylation of a } \\
\text { number of loci including } \mathrm{MLH1}\end{array}$ & $\begin{array}{l}\text { WGS showed that among } 30 \text { hypermutated tumors, } 17 \\
\text { had CIMP }\end{array}$ & 3,23 \\
\hline MYH or MUTYH & DNA base excision repair & $\begin{array}{l}\text { WES identified } 6 \text { CRC tumors and } 1 \text { CRC adenoma } \\
\text { harboring germline } M Y H \text { mutations with increased } \\
\text { mutational load }\end{array}$ & 24 \\
\hline POLE & $\begin{array}{l}\text { DNA polymerase involved in } \\
\text { leading strand synthesis and base } \\
\text { excision repair }\end{array}$ & $\begin{array}{l}\text { WGS identified tumors harboring germline or sporadic } \\
\text { mutations in POLE associated with ultramutated } \\
\text { phenotype }\end{array}$ & 8,9 \\
\hline POLD1 & $\begin{array}{l}\text { DNA polymerase involved in DNA } \\
\text { synthesis and repair }\end{array}$ & $\begin{array}{l}\text { WGS identified tumors harboring germline or sporadic } \\
\text { mutations in POLD1 associated with ultramutated } \\
\text { phenotype }\end{array}$ & 8,9 \\
\hline$T G F-\beta R I I$ & Regulation of cell growth & $\begin{array}{l}\text { Human colon cancer cell lines with high rates of MSI were } \\
\text { associated with mutations in TGF- } \beta \text { RII via automated } \\
\text { sequencing }\end{array}$ & 25 \\
\hline ACVR2A, APC, SLC9A9, TCF7L2 & $\begin{array}{l}\text { Various functions in regulating cell } \\
\text { growth, division, adhesion, and } \\
\text { transport }\end{array}$ & $\begin{array}{l}\text { WGS identified hypermutated tumors frequently } \\
\text { associated with these mutations }\end{array}$ & 3 \\
\hline TCF4, IGF2R, BAX & $\begin{array}{l}\text { Various functions in regulating } \\
\text { cell growth, division, and } \\
\text { differentiation }\end{array}$ & $\begin{array}{l}\text { Large panel sequencing identified MSI CRC tumors } \\
\text { commonly associated with these mutations }\end{array}$ & 5 \\
\hline
\end{tabular}

Abbreviations: CIMP, CpG island methylator phenotype; CRC, colorectal cancer; MMR, mismatch repair; MSI, microsatellite instability; PCR, polymerase chain reaction; WES, whole-exome sequencing; WGS, whole-genome sequencing. 
to PD-1 blockade, particularly as more cases with POLE mutations are identified. Future studies should also explore the immune profile of POLE-mutated $\mathrm{mCRC}$ in comparison to MSI-H mCRC. These will be more readily achievable given the continued development of comprehensive genomic profiling via massive parallel sequencing, which has already dem- onstrated high concordance with MSI status and reliable detection of MSI based on mutational load. ${ }^{12,21}$ Future studies will also likely focus on investigating numerous molecular profiles (Table 1) associated with a hypermutated phenotype or defective MMR that may similarly predict benefit to immune checkpoint blockade. . $^{3,5,8,9,22-25}$

\section{References}

1. Siegel RL, Miller KD, Jemal A. Cancer statistics, 2016. CA Cancer J Clin 2016;66:7-30.

2. Gong J, Cho M, Fakih M. RAS and BRAF in metastatic colorectal cancer management. J Gastrointest Oncol 2016;7:687-704.

3. Cancer Genome Atlas Network. Comprehensive molecular characterization of human colon and rectal cancer. Nature 2012;487:330337.

4. Le DT, Uram JN, Wang H, et al. PD-1 blockade in tumors with mismatchrepair deficiency. N Engl J Med 2015;372:2509-2520.

5. Lin EI, Tseng LH, Gocke CD, et al. Mutational profiling of colorectal cancers with microsatellite instability. Oncotarget 2015;6:42334-42344.

6. Le DT, Uram JN, Wang H, et al. Programmed death-1 blockade in mismatch repair deficient colorectal cancer [abstract]. J Clin Oncol 2016;34(Suppl):Abstract 103.

7. Overman MJ, Kopetz S, McDermott RS, et al. Nivolumab \pm ipilimumab in treatment (tx) of patients (pts) with metastatic colorectal cancer (mCRC) with and without high microsatellite instability (MSI-H): CheckMate-142 interim results [abstract]. J Clin Oncol 2016;34(Suppl):Abstract 3501

8. Palles C, Cazier JB, Howarth KM, et al. Germline mutations affecting the proofreading domains of POLE and POLD1 predispose to colorectal adenomas and carcinomas. Nat Genet 2013;45:136-144.

9. Briggs $S$, Tomlinson I. Germline and somatic polymerase $\varepsilon$ and $\delta$ mutations define a new class of hypermutated colorectal and endometrial cancers. J Pathol 2013;230:148-153.

10. Kothari N, Teer JK, Abbott AM, et al. Increased incidence of FBXW7 and POLE proofreading domain mutations in young adult colorectal cancers. Cancer 2016;122:2828-2835.

11. Domingo E, Freeman-Mills L, Rayner E, et al. Somatic POLE proofreading domain mutation, immune response, and prognosis in colorectal cancer: a retrospective, pooled biomarker study. Lancet Gastroenterol Hepatol 2016;1:207-216.

12. Stadler ZK, Battaglin F, Middha S, et al. Reliable detection of mismatch repair deficiency in colorectal cancers using mutational load in nextgeneration sequencing panels. J Clin Oncol 2016;34:2141-2147.

13. Bellone $S$, Centritto F, Black J, et al. Polymerase $\varepsilon$ (POLE) ultra-mutated tumors induce robust tumor-specific CD4+ $\mathrm{T}$ cell responses in endometrial cancer patients. Gynecol Oncol 2015;138:11-17.
14. van Gool IC, Eggink FA, Freeman-Mills L, et al. POLE proofreading mutations elicit an antitumor immune response in endometrial cancer. Clin Cancer Res 2015;21:3347-3355.

15. Howitt BE, Shukla SA, Sholl LM, et al. Association of polymerase e-mutated and microsatellite-instable endometrial cancers with neoantigen load, number of tumor-infiltrating lymphocytes, and expression of PD-1 and PD-L1. JAMA Oncol 2015;1:1319-1323.

16. Llosa NJ, Cruise M, Tam A, et al. The vigorous immune microenvironment of microsatellite instable colon cancer is balanced by multiple counterinhibitory checkpoints. Cancer Discov 2015;5:43-51.

17. Rizvi NA, Hellmann MD, Snyder A, et al. Cancer immunology. Mutational landscape determines sensitivity to PD-1 blockade in non-small cell lung cancer. Science 2015;348:124-128.

18. Mehnert JM, Panda A, Zhong $\mathrm{H}$, et al. Immune activation and response to pembrolizumab in POLE-mutant endometrial cancer. J Clin Invest 2016;126:2334-2340.

19. Santin AD, Bellone S, Buza N, et al. Regression of chemotherapy-resistant polymerase epsilon (POLE) ultra-mutated and MSH6 hyper-mutated endometrial tumors with nivolumab. Clin Cancer Res 2016;22:5682-5687.

20. Glaire MA, Domingo E, Vermeulen L, et al. POLE proofreading domain mutation defines a subset of immunogenic colorectal cancers with excellent prognosis [abstract]. Ann Oncol 2016;27(Suppl 6):Abstract 4600.

21. Hall MJ, Gowen K, Sanford EM, et al. Evaluation of microsatellite instability (MSI) status in gastrointestinal (GI) tumor samples tested with comprehensive genomic profiling (CGP) [abstract]. J Clin Oncol 2016;34(Suppl 4):Abstract 528.

22. Link JT, Overman MJ. Immunotherapy progress in mismatch repairdeficient colorectal cancer and future therapeutic challenges. Cancer J 2016;22:190-195.

23. Levine AJ, Phipps AI, Baron JA, et al. Clinicopathologic risk factor distributions for MLH1 promoter region methylation in CIMP-positive tumors. Cancer Epidemiol Biomarkers Prev 2016;25:68-75.

24. Jones S, Emmerson P, Maynard J, et al. Biallelic germline mutations in MYH predispose to multiple colorectal adenoma and somatic G:C.->T:A mutations. Hum Mol Genet 2002;11:2961-2967.

25. Markowitz S, Wang J, Myeroff L, et al. Inactivation of the type II TGFbeta receptor in colon cancer cells with microsatellite instability. Science 1995;268:1336-1338. 